\title{
BUILDERS OF THE FUTURE
}

\section{O'ZBEK TILSHUNOSLIGIDA FRAZEOLOGIK BIRLIKLARNING TURKUMLANISHI}

\section{Gulbahor Mirzajonova}

Farg'ona Davlat Universiteti filologiya fakulteti o'zbek tili yo'nalishi talabasi Nabiyeva Gulyora

Farg'ona Davlat Universiteti filologiya fakulteti o'zbek tili yo'nalishi talabasi

\section{DOI - https://doi.org/10.37547/builders-07}

Annotatsiya: Ushbu maqolada O'zbek tilshunosligida frazeologik birliklarning turkumlanishi. Ma'no yaxlitligiga ega bo' Igan va nutqiy jarayonga qadar ikki va undan ortiq so'zlarning barqaror munosabatidan tashkil topgan, nutqga tayyor holda olib kiriluvchi ko'chma manodagi barqaror birikmalarga frazeologizmlar deyiladi.

Kalit so'zlar: O'zbek tilshunosligi, frazeologik birliklar, nutqiy jarayon, barqaror munosabat, nutq, ko'chma mano, frazeologizmlar.

KIRISH Frazeologizmlar- ma'nosi bir so'zga teng keladigan gap yoki so'z birikmalaridir.

Masalan: Xamirdan qil sug'urganday iborasi oson so'ziga teng keladi, bir yoqadan bosh chiqarib- ahil bo'lib; ko'ngli joyiga tushdi- tinchidi; tepa sochi tikka bo'ldi- jahli chiqdi; tekkanga tegib tegmaganga kesak otadi- xira tegajoq; qo'y og'zidan cho'p olmagan - yuvosh; yerga ursa ko'kka sakraydi-juda sho'x; og'zining tanobi qochmoq- quvonmoq kabi.

Frazeologiyaning o'rganish predmeti frazeologizmning tabiati va substansional xususiyatlari hamda ularning nutqda amal qilish qonuniyatlaridir.

Frazeologizm lisonda barcha lisoniy birliklarga xos bo'lgan umumiylik tabiatiga ega va nutqda xususiylik sifatida namoyon bo'ladi.

Frazeologizmlar lug'aviy birlik bo'Iganligidan u nutq jarayonida gap tarkibida bir mustaqil so'z kabi harakat qiladi bir gap bo'lagi yoki kengaytiruvchi sifatida keladi: Masalan: Rais daladagi hosilni ko'rib boshi osmonga yetdi. Bu gapda boshi osmonga yetdi iborasi butun holda murakkab kesim vazifasini bajargan.

Frazeologizmlar, asosan, belgi va harakat ifodalaydi. Ularning ma'nosi bir so'zga teng keladi, shuning uchun iboraning turkumi u teng kelgan so'zning turkumiga qarab olinadi. Fe'l turkumiga mansub frazeologizmlar:me'dasiga tegmoq, yaxshi ko'rmoq, holdan toymoq, sabr kosasi to'Imoq, tepa sochi tikka bo'Imoq, og'ziga talqon solmoq, podadan oldin chang chiqarmoq. 


\section{BUILDERS OF THE FUTURE}

Sifat turkumiga mansub frazeologizmlar :

Ko'ngli bo'sh, rangi sovuq, yuragi toza, avzoyi bejo, dili siyoh, kayfi buzuq.

Ravish turkumiga mansub frazeologizmlar:

Ipidan-ignasigacha, ikki dunyoda ham, miridan-sirigacha, ha-hu deguncha.

So'z-gaplarga mansub frazeologizmlar:

Turgan gap, shunga qaramay, katta gap.

Gap tarkibida iboralar o'zi teng kelgan so'zning so'rog'ini oladi va yaxlit holda bitta gap bo'lagi vazifasida keladi. Ibora so'z birikmasi yoki gap singari nutq jarayonida hosil bo'Imaydi, balki xuddi leksema kabi tilda tayyor holda bo'ladi. Demak, ibora nutq hodisasi emas, balki lisoniy birlikdir.

Iboradan anglashilgan belgi, miqdor va harakat kabilar haqidagi ma'lumot frazeologik ma'no (iboraning atash semasi) deyiladi. Iboraning atash semasi biror leksemaning atash semasiga teng.

\section{Xullosa}

Frazema til qurilishining lug'at bosqichiga mansub ikkinchi lisoniy birlik bo'lib, bittadan ortiq leksemaning o'zaro semantik-sintaktik birlashuvi bilan tarkib topgan bo'ladi. Shunga ko'ra tuzma semantik birlik deyiladi. Sintaktik tuzilishi jihatdan birikmaga, gapshaklga teng bo'lib, odatda nominativ va sinifikativ vazifa bajaradi. Frazeologizmlarning ifoda jihati deb ularning asli qanday lisoniy biroiklardan tarkib topgani tushuniladi. Frazema ikki va undan ortiq leksemaning o'zaro semantik-sintaktik bog'lanib, umumlashma ko'chma mano kashf etishi bilan yuzaga keladi.

\section{Adabiyotlar:}

Sayfullayeva R., Mengliyev B., Boqiyeva G., Hozirgi o'zbek adabiy tili- Toshkent : Fan va texnologiyalar, 2010.

2 Lutfullayeva D., Davlatova R., Saparniyazova M., Hozirgi o'zbek adabiy tili - Toshkent: "Iqtisod-Moliya", 2018.

3. Rahmatullayev Sh. Hozirgi adabiy o'zbek tili, - Toshkent : "Universitet", 2006.

4. Nurmonov Abdulhamid O'zbek tilshunoslik tarixi- Toshkent : "O'zbekiston", 2002.

5. Rasulov R. Umumiy tilshunoslik - Toshkent : Fan va texnologiyalar, 2010. 